\title{
A Study of Service Desk Setup in Implementing IT Service Management in Enterprises
}

\author{
Xiaojun Tang', Yuki Todo ${ }^{2}$ \\ ${ }^{1}$ Enterprise Key Account IT Service Department, Shanghai Wicresoft Co., Ltd., Shanghai, China \\ ${ }^{2}$ Department of Electrical and Computer Engineering, Kanazawa University, Kanazawa, Japan \\ Email: tony_txj@hotmail.com,yktodo@se.kanazawa-u.ac.jp
}

Received May 13, 2013; revised June 13, 2013; accepted June 20, 2013

Copyright (c) 2013 Xiaojun Tang, Yuki Todo. This is an open access article distributed under the Creative Commons Attribution License, which permits unrestricted use, distribution, and reproduction in any medium, provided the original work is properly cited.

\begin{abstract}
Information Technology Service Management (ITSM) provides a framework to provide IT related services and the interaction of IT staff with users, and is often related with the British Government's Information Technology Infrastructure Library (ITIL). ITIL offers a set of "best practices" for managing IT services and is one of the most widely accepted approaches to IT service management in the world. Recently, more and more enterprises implemented a centralized IT service management model based on the ITIL framework. However, even by adopting ITIL, most of the enterprises didn’t improve their IT service management level. Some factors become the barriers to the success of ITIL and ITSM implementation. In some cases, enterprises paid more attention to IT infrastructure setup and operation but neglect the importance of Service Desk. In this study, we first review ITSM, ITIL and Service Desk. Second, we identify a full function of new Service Desk and how to set up a good Service Desk. Enterprises should take proper measures in building a mature Service Desk to ensure successful implementation of ITSM.
\end{abstract}

Keywords: Information Technology Service Management (ITSM); Information Technology Infrastructure Library (ITIL); Service Desk

\section{Introduction}

In today's demanding world, IT plays a pivotal role in creating new opportunity and delivering competitive advantage to enterprises. Business focused approaches on managing IT resources are highly valued in today's enterprise. As IT departments and organizations transition from technology to service based management models, investing in a credible, best practice framework such as Information Technology Infrastructure Library (ITIL) is becoming more important [1].

An important aspect of the new legislation is the requirement for enterprises to have consistent and formalized application controls as well as general controls across all IT functions, including areas outsourced to external partners. To achieve these goals, organizations are adopting ITIL to provide a framework and implement IT service management in enterprise [2].

According to the previous research about the ITIL/ ITSM application status concluded in Taiwan, the major challenges of implementing ITIL as listed in Table 1. The top five challenges are culture, integration with current process, ITIL/ITSM related knowledge, appropriate management tool, and clear measurement target [3,4].

After reviewing the top five challenges, culture shift, appropriate management tool and clear measurement target are all directly related to Service Desk. Also, Service Desk, as the single point of contact, will be very useful for culture shift and ITIL/ITSM related knowledge popularize. Thus, a good Service Desk is very important in successfully implementing ITIL/ITSM in enterprises [5].

Table 1. Major challenges of implementing ITIL (Source: KPMG 2007).

\begin{tabular}{cc}
\hline Major challenges & Percentage \\
\hline Culture shift & $17.2 \%$ \\
Integration with current process & $15.5 \%$ \\
ITIL/ITSM related knowledge & $13.8 \%$ \\
Appropriate management tool & $10.3 \%$ \\
Clear measurement target & $10.3 \%$ \\
Project within budget & $8.6 \%$ \\
Insufficient internal professional staff & $8.6 \%$ \\
Project on time & $5.2 \%$ \\
Managing consultants & $1.7 \%$ \\
Incapable and inexperienced consultants & $1.7 \%$ \\
\hline
\end{tabular}


Usually, the Service Desk concentrates on incident lifecycle management and performs the following primary functions [6]:

- Customer Interface.

- Business Support.

- Incident Control.

- Management Information.

Service Desk is the primary point of contact for all internal and/or external customers. It will be the function which users are most easily perceived. Users' impressions of Service Desk impact the image of enterprises' IT service a lot. A good image of Service Desk can help IT department obtain more support for senior management board and business department, which will be very helpful to propel ITSM implementation and get higher customer satisfaction.

In the past, the goal of a traditional Service Desk is only to restore the service as quickly as possible, manage the incident life-cycle (coordinating resolution) and generate reports, communicate and promote. It handles calls, questions, requests, complaints and remarks [7]. But now, enterprises need a Service Desk with more functions and stronger capabilities. A good and integral Service Desk will be very important in implementing ITSM. In this article, we first offer a review of ITSM/ITIL, and the definition of Service Desk, present the importance of Service Desk, then, discuss the new functions of Service Desk. Finally, some suggestions about how to set up a good Service Desk via three different perspectives, process, people and tool, are discussed.

\section{Literature Review}

\subsection{ITSM}

IT service management (ITSM) refers to the implementation and management of quality IT services that meet the needs of the business. IT service management is performed by IT service providers through an appropriate mix of people, process and information technology. The following represents a characteristic statement from the ITSM literature [8]:

"Providers of IT services can no longer afford to focus on technology and their internal organization; they now have to consider the quality of the services they provide and focus on the relationship with customers."

Therefore, the providers of IT services should shift their focus on technology and internal organization to the quality of services they provides and the customers' satisfaction. If the IT services providers cannot provide proactive and value-added service to client and end users, we can say the IT service level is low, although the IT services provider have a huge infrastructure with latest technologies.

IT department must try to attain the proactive level of IT service management process maturity (see Figure 1) and successfully deploy these emerging ITSM technologies $[9,10]$.

\subsection{ITIL}

The Information Technology Infrastructure Library (ITIL), which was developed by OGC (Office of Government

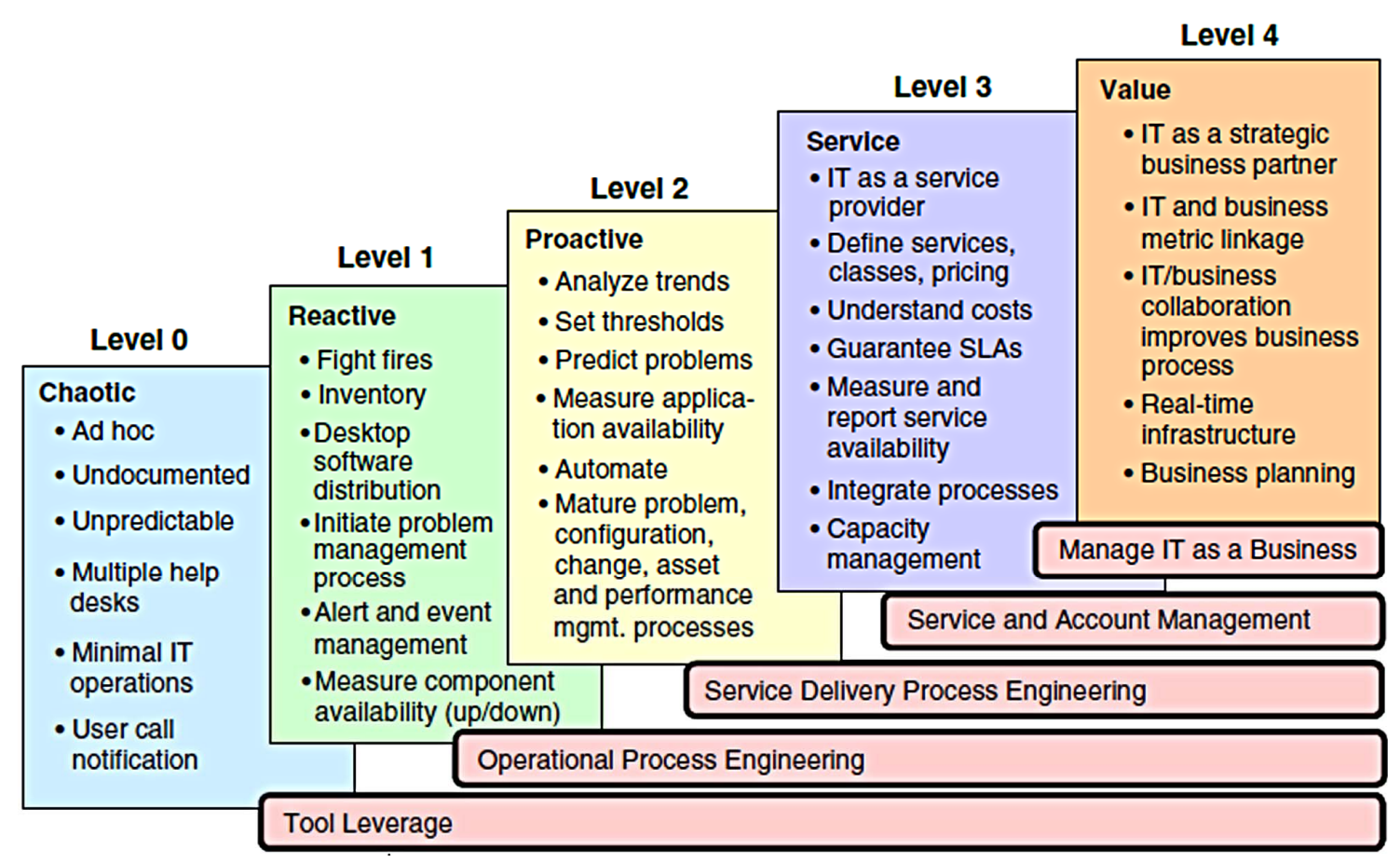

Figure 1. IT service management maturity model. 
Commerce) of UK government, offers a detailed description of "best practices" for IT service management [11]. The goal of ITIL is to provide a framework of best practices that organizations can use as a guideline for managing their IT organizations. Five core titles are included in ITIL v3, which are:

- Service Strategy.

- Service Design.

- Service Transition.

- Service Operation.

- Continual Service Improvement.

\subsection{The Definition of Service Desk in ITIL}

Service Desk is a primary IT service called for in IT service management (ITSM) as defined by the Information Technology Infrastructure Library (ITIL). It is intended to provide a Single Point of Contact (SPOC) to meet the communication needs of both Users and IT employees, but also to satisfy both Customer and IT Provider objectives. Service desk handles incidents and service requests, as well as providing an interface to users for other ITSM activities such as:

- Incident management.

- Problem management.

- Configuration management.

- Change management.

- Release management.

- Service-level management.

- Availability management.

- Capacity management.

- Financial management.

- IT service continuity management.

- Security management.

Mission of Service Desk is to be a central point of contact between the User and IT Service Provider [11].

\subsection{The Definition of Service Desk in COPC}

In COPC's view, the main responsibilities of IT Service Desk are:

- Accept customer request (via telephone, e-mail, fax and etc.).

- Record and track incident and users' feedback.

- Update users the current status and progress about their request in a timely manner.

- Primarily evaluating users' request according to the Service Level Agreement, try to resolve it or assign to the related persons.

- Monitor the implementation of policies according to the Service Level Agreement, propose to revise policies if necessary.

- Manage the life-cycle of each service request.

- Communicate with users if Service Level Agree is adjusted.
- Coordinate 2nd tier and 3rd tier support team.

- Provide management related information and suggestion, improve service quality.

- Discover the problem in IT Service operation from users' feedback.

- Discover users' requirement of training and education.

- Terminate incident and confirm resolution with users.

\section{New Service Desk Functions}

\subsection{Benefit of New Service Desk}

A traditional Service Desk only plays a contact point in IT service organization, but enterprises expect it brings more value and benefits. A good Service Desk provides the following benefits to enterprises:

1) Direct Benefits

a) Reduced cost of incident resolution

b) Reduced self-inflicted incidents via integrated and reliable change

c) Increased productivity of IT staff

d) Improved asset utilization, life cycle management and accurate software licensing costs

e) Reduced service cycle times

f) End-to-end service cost optimization

2) Indirect Benefits

a) Reduced peer support

b) Standardization

c) Consolidation

d) Non-IT staff more productive

e) Improved availability

f) Managing appropriate expectations

g) Improved efficiency of security and business continuity planning processes

h) Improved IT governance

i) Drives continual improvement

Thus, when implementing ITSM in enterprise, the responsibilities and goals should be adjusted to realize the above benefits.

\subsection{New Service Desk Functions}

With the development of technology and service requirement, the position of Service Desk is changed. It plays an important role in Service Operation (see Figure 2).

Service Desk needs not only accept the service request reactively, but also discover the problem proactively [12]. The suggested functions of new Service Desk are:

- Filter and categorize service requests.

As the SPOC of IT service, Service Desk accepts all the service requests from end users. Most of the service requests are only to request related information or resolve easy problems. That's to say, most of the service request will be resolved and closed by Service Desk at 


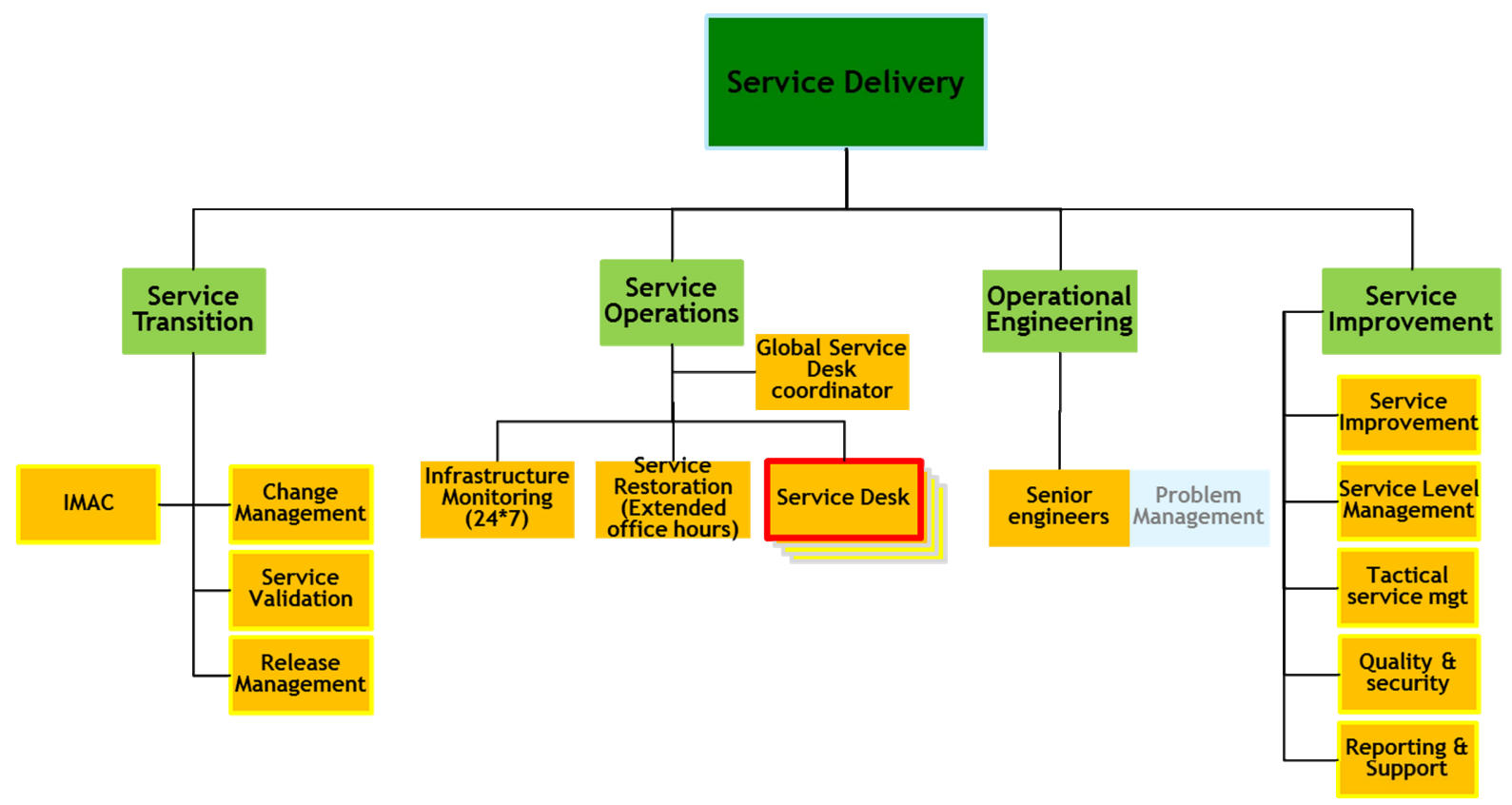

Figure 2. Position of service desk in organization.

the first contact. Usually, more than $60 \%$ service requests are resolved by Service Desk directly, then they categorize the rest requests and assign to the appropriate 2nd tier or 3rd tier support team. Service Desk plays the most important role in IT department, they handle most of the requests from the end users, and also be the interface to contact with the backend team [13].

- In charge of Incident Management process

Incident management can be defined as an unplanned interruption to an IT Service or a reduction in the Quality of an IT Service. The objective of incident management is to restore normal operations as quickly as possible with the least possible impact on either the business or the user, at a cost-effective price $[7,8]$.

Service Desk should be the owner of Incident Management process, they are primary focus to ensure a prompt recovery of the system, supervising and directing the internal or external resources.

- Knowledge Base Maintenance

Knowledge Base $(\mathrm{KB})$ is a special kind of database for knowledge management. A knowledge base is an information repository that provides a means for information to be collected, organized, shared, searched and utilized. It can be either machine-readable or intended for human use.

Service Desk is the key user of Knowledge Base. In daily work, they can easily know which KB article is useful, which one is out of date and which one has very low resolve rate. With such information records, Service Desk should proactively maintain KB. For example, they can promote a $\mathrm{KB}$ article due to its high resolve rate, propose to update or expire an article due to it is out of date or quite low resolve rate. With a strong KB, Service Desk can improve their work efficiency and resolve the issue for end users more effective.

- Initiative Problem Management process

Service Desk also should play an important role in Problem Management process. Problem can be collected from incidents. Service Desk should have the awareness to define a problem in a timely manner. For example, if Service Desk received a lot of incidents with similar symptom, they should consult the senior technical staff to conduct Root Cause Analysis (RCA) process as soon as possible. Once a problem is defined timely, incidents can be linked to the problem, and then, Service Desk can inform end users timely about the resolution or work around $[7,8]$.

- Coordinator of Change Management Process

The objective of change management is to ensure that standardized methods and procedures are used for efficient and prompt handling of all changes to control IT infrastructure, in order to minimize the number and impact of any related incidents upon service. Usually, change requests need to be reviewed and approved by several peoples for impact and risk analysis, roll back plan, $C A B$ confirm and etc. Urgent change request also has its different approval process [7-9].

From Service Desk view, they are not only responsible to log the change request, but also need to track and push on the progress, ensure the standardized methods and procedures are used for efficient and prompt handling of all changes.

- Coordinate onsite service and VIP support

For that part of the incidents and service requests where 
the Service Desk is not able to execute the service remotely, On-site Support Services will be used to execute the service onsite, such as hardware replacement. In this case, Service Desk should still be the primary contact point of end users. They are responsible for giving end users an expectation about when the onsite staff will arrive and when the issue can be fixed. Moreover, a lot of enterprise has VIP support process to ensure the high service level for VIP. Service Desk also needs to communicate with VIP timely for any information updated and collects feedback.

- Event Management (Proactive Monitoring Service)

Event Management is the process that monitors all events that occur through the IT infrastructure. It allows for normal operation and also detects and escalates exception conditions.

Service Desk cannot only accept request reactively, another function for them is providing proactive monitoring service. Service Desk can choose appropriate tool to monitor the status of servers, network devices, storage, UPS and etc. If any alert is received, Service Desk generates an incident, problem or change timely and process into the related ITSM process, fixes the potential issue and ensures the IT infrastructure work in a healthy status [9].

- Responsible for Reporting Service

Reporting Service reflects the status in IT service operation. An accurate and timely report is very important for IT operation service review and forecast. A good Service Desk is able to handle the reporting service, as they handle almost all requests and know which need to be emphasized [14]. They make sure the report reflect the actual status and help to find the weakness to be improved.

- Customer Satisfaction Survey

Currently, most of the ITSM tool has the function to conduct customer satisfaction survey automatically; this did save time and improved efficiency. However, the same questionnaire cannot collect the different voices from end users, even not to mention how many end users will complete the template survey carefully. Service Desk should have a process to pick up some service requests and call back the end users to learn their feedback. Such straightforward way is very useful to understand our customers' real needs, which direct IT department to improve their service [15].

\section{Setup a Great Service Desk}

Obviously, a traditional Service Desk cannot meet the new requirements of enterprises. When implementing ITSM, we should consider setup a Service Desk with full functions. When setting up the new Service Desk, we can consider from three different perspectives, process, people and tool.

\subsection{Process}

Process is the most important part in Service Desk, this ensure everyone work in the same guideline and using the same language. When designing the process, we should focus on the ability to efficiently provide clients and end users with services that meet their expectations, also focuses on the mechanisms to quantitatively evaluate, maintain, and improve these processes to ensure they function effectively and efficiently [16].

There are 14 key service desk processes including: service desk dashboard, operate service desk process, communicating with customers, promote and market service desk, managing costs and recovery process, preparing reports process, monitoring process, optimize service desk process, determining outsourcing requirements, optimizing staff levels process, optimizing staff skills process, optimizing technology process and reviewing and optimizing monitoring and reporting [11].

When designing the process, the following parts need to be taken into consideration [17].

- How to implementing new services, programs, clients, and requirements

Process must design to ensure Service Desk implements new services, programs, clients, and requirements efficiently meet client and end-user requirements and targets.

\section{- Process capability}

Process must ensure Service Desk is capable of consistently achieving targets. Process needs to be adjusted if Service Desk failure to achieving targets in continuous evaluation periods.

- Corrective action and continuous improvement

Service Desk should use a structured approach for identifying and resolving the root cause of poor performance or the performance metrics not consistently meeting requirements and targets. The approaches should be recorded to track the effectiveness and for further usage.

\section{- Transaction monitoring}

Service Desk must have an approach for monitoring transactions that is designed to meet client, and end-user requirements and targets. This approach must focus on identify and correct program-level issues that cut across multiple Service Desk agents at the process level [17].

- Data collection, analysis and action

Service Desk must have a process to collect, analyze, and use performance data to achieve its customer satisfaction, service, quality, cost, and employee performance targets, also the process must ensure that the data are collected, usable, integrity, known by appropriate personnel and also lead to actions if results fall below the targets.

- End-user privacy

The process must protect end-user sensitive and proprietary data and information. 


\subsection{People}

Meeting performance targets and improving performance levels requires a workforce that is appropriately skilled and motivated. Service Desk should have people management approaches that enable all staff to effectively and efficiently deliver quality services. When managing the Service Desk staffs, the following points can be taken into consideration $[18,19]$.

- Defining jobs, recruiting and hiring

Service Desk must have clear, written definitions of the minimum skills and knowledge required for Service Desk jobs, job definition can refer to the Service Desk functions, and those jobs that include training delivery and monitoring transactions. Service Desk must acquire staffs which have a high probability of successfully performing their jobs.

- Training and development

Service Desk must provide the training and development that all staff performing jobs needs to acquire and maintain the skills and knowledge required for their positions. Trainings need to be adjusted by considering different attendees' level, positions and capabilities.

- Verifying skills and knowledge

To ensure that all staff has maintained the necessary skills to successfully perform their jobs, Service Desk should verify the skills and knowledge of all staff on a yearly basis. Only qualified staff can continue to serve customers. This also should be included in performance appraisal.

- Staff performance management

Service Desk must have a performance evaluation system to monitor and encourage staff. Analysis also should be conducted in order to work out follow-up action items to continuously improve performance $[18,19]$.

- Managing staff feedback

Service Desk must use a structured approach for proactively soliciting, evaluating, and taking appropriate action on feedback obtained from individuals.

\subsection{Tool}

The tools for Service Desk should be designed for collecting consistent, time sensitive, and documented service request report data. Currently, many of Service Desk products include features to automate the approval process of an incident report or case investigation. These products should also have the ability to collect real time incident information such as time and date data. Additionally the tool should automatically send notifications, assign tasks and escalations to appropriate individuals depending on the incident type, priority, time, status and custom criteria. Currently, a lot of products provide the ability for administrators to configure the report forms as needed, create analysis reports and even set access controls on the data.
For most of Service Desk functions requirement in enterprises, Remedy from BMC software is widely used. It has been used by more than $90 \%$ of TOP500 companies. It enables comprehensive, best practice based incident management and problem management processes via intuitive, easily adopted, enterprise scale technology.

As for the proactive monitoring service requirement in Service Desk, Microsoft System Center is also widely used in enterprises. System Center Operation Manager (SCOM), as one of the most important component of System Center, provides deep visibility into the health, performance, and availability of IT infrastructure-across applications, operating systems, hypervisors and hardware-through a single interface. When choosing the appropriate tool for monitoring purpose, it is highly recommended to choose a centralized system monitoring platform that's flexible and cost-effective, helps ensure the predictable performance and availability of vital applications, and offers comprehensive monitoring for infrastructure.

General speaking, when implementing ITSM in enterprise, IT department needs to find the most suitable tool according to the functions of Service Desk. The capability of the selected tool must meet all the requirement of Service Desk and another important thing is, easy to use.

\subsection{Case Study}

Here is a case study in Nanjing, China. Before 2010, the service desk applied a traditional process and functions. They received service requests reactively, and then category the service requests into different genres and dispatch to the related team. However, the result is not good due to the following reasons:

- Service Desk engineers' skill cannot be improved by handling real tickets, which caused high attrition rate and higher training cost.

- 2nd tier technical engineer spent a lot of time in replying easy questions and inquiry, cannot focus on the technical part, the work efficiency is low.

- There is no a centralize method to manage and update knowledge, the resolve rate mostly depended on experience.

- Service desk didn't have the awareness to be involved in the Problem/Change management process.

- VIPs' requests cannot be addressed timely.

- Cannot proactively find the potential issues

To improve the service level of service desk, the service desk was restructured in 2010. The major change was responsibilities. Besides Incident Management process, the new service desk was assigned to be involved into more of ITIL process, such as: Problem Management, Change Management, Event Management, Service Level Management and etc. With about 6 months' efforts, the restructured was completed successfully. After 2 years 
Table 2. Service Desk KPI Improved.

\begin{tabular}{ccc}
\hline KPI & $\mathbf{2 0 0 9}$ & $\mathbf{2 0 1 1}$ \\
\hline Customer satisfaction & $71 \%$ & $81 \%$ \\
VIP satisfaction & $60 \%$ & $90 \%$ \\
Average handle time & $15 \mathrm{~min}$ & $12 \mathrm{~min}$ \\
Call abandon rate & $10 \%$ & $3 \%$ \\
SD help resolve rate & $\mathrm{N} / \mathrm{A}$ & $60 \%$ \\
Staff attrition rate & $20 \%$ & $8 \%$ \\
Problem identify & 4 & 7 \\
Knowledge base usage & $30 \%$ & $80 \%$ \\
Change execute time & 8 days & 5 days \\
\hline
\end{tabular}

operations, the KPI (Key Performance Indicators) had been improved apparently (Table 2).

\section{Conclusions}

As several key successful factors to succeeding in ITSM implementation, we suggest that enterprise attach importance to Service Desk. Service Desk can be implemented in many different ways [3]; however by following the best practices outlined in ITIL, it should meet the most important need of the end user and enterprise. In addition, by following the best practices, the Service Desk will enable the enterprise to have a foundation for the IT department not only to meet the increasing needs of the end user, but for the IT department to link into strategic areas within the company [6].

A good Service Desk allows enterprise to be less dependent on specific technology and enables them to easily connect with business partners by switching the ITSM implementation focus on the infrastructure delivers value adding to the corporate users and customers. A good Service Desk improves a company's customer satisfaction by allowing the company to implement their ITSM even business plan. A good Service Desk communicates effectively and efficiently with each end user. There are many more things which a good Service Desk can do well, so let's pay more attention on Service Desk when implementing ITSM.

\section{REFERENCES}

[1] C. Zhao, H. H. Gan and F. Gao, "A Study on the Process Model for IT Service Management,” Proceedings of Finite Element Analysis and CAD, Peking University Press, Beijing, 1994, pp. 1-5.

[2] J. van Bon, M. Picper and A. der Veen, "Foundations of IT Service Management Based on ITIL,” 2nd Edition, Van Haren Publishing, Zaltbommel, 2005.

[3] W. G. Tan, A. Cater-Steel and M. Toleman, "Implementing IT Service Management: A Case Study Focusing on Critical Success Factors," Journal of Computer Information Systems, Vol. 50, No. 2, 2009, pp. 1-12.

[4] A. Chen and S. K. Chou, "Issue in Implementing Infor- mation Technology Service Management” Service Science, Vol. 1, No. 2, 2010, pp. 1-5.

[5] J. P. Wan, S. Q. Zhu and Y. F. Wang, "Empirical Analysis on Risk Factors of IT Service Management Project Implementation," IEEE International Conference on Wireless Communications, Networking and Mobile Computing, Dalian, 14-16 October 2008, pp. 1-4.

[6] R. A. Steinberg, “ITIL Service Operation,” 2011 Edition, Randy A. Steinberg, Trafford, 2011.

[7] J. van Bon, M. Picper and A. der Veen, "Foundations of IT Service Management Based on ITIL," 2nd Edition, Van Haren Publishing, Zaltbommel, 2005.

[8] Official ITIL Website, “IT Service Management,” 2013. http://www.itil-officialsite.com/home/home.aspx

[9] D. Clifford and J. van Bon, "Implementing ISO/IEC 20000 Certification: The Roadmap. ITSM Library,” David Clifford, Jan van Bon, 2008.

[10] J. P. Wan and D. Wan, “Analysis on the Mindbugs in Information Technology Service Management Project Implementation,” Technology and Investment, Vol. 2, No. 1, 2011, pp. 184-192. doi:10.4236/ti.2011.23019

[11] Wikipedia, "Information Technology Infrastructure Library,” 2013. http://en.wikipedia.org/wiki/ITIL

[12] M. Toleman, A. Cater-Steel, B. Kissell, R. Chown and M. Thompson, "Improving ICT Governance: A Radical Restructure Using CobiT and ITIL,” In: A. Cater-Steel, Ed., Information Technology Governance and Service Management: Frameworks and Adaptations, Information Science Reference, Hershey, 2009, pp. 178-189.

[13] A. Cater-Steel and W. Tan, "The Role of IT Service Management in Green IT,” Australasian Journal of Information Systems, Vol. 17, No. 1, 2010, pp. 3-15.

[14] C. P. Praeg and D. Spath, "Perspectives of IT-Service Quality Management: A Concept of Life-Cycle Based Quality Management of IT Services,” In: A. Cater-Steel, Ed., Information Technology Governance and Service Management: Frameworks and Adaptations, Information Science Reference, Hershey, 2009, pp. 381-407.

[15] A. Cater-Steel, W. Tan and M. Toleman, "Summary of ITSM Standards and Frameworks Survey Responses," Proceedings of the itSMF Australia 2008 Conference, 27-29 August 2008, pp. 2-10.

[16] J. P. Wan, H. Zhang and D. Wan, "Evaluation on Information Technology Service Management Process with AHP,” Technology and Investment, Vol. 2, No. 1, 2011, pp. 38-46. doi:10.4236/ti.2011.21005

[17] F. F. Reichheld and W. E. Sasser, "Zero Defections: Quality Comes to Services,” Harvard Business Review, Vol. 68, No. 5, 1990, pp. 105-111.

[18] O. O. Sawyerr, S. Srinivas and S. Wang, "Call Center Employee Personality Factors and Service Performance," Journal of Services Marketing, Vol. 23, No. 5, 2009, pp. 301-317. doi:10.1108/08876040910973413

[19] J. Hogan and B. Holland, "Using Theory to Evaluate Personality and Job-Performance Relations: A Socioanalytic Perspective,” Journal of Applied Psychology, Vol. 88, No. 1, 2003, pp. 100-112. doi:10.1037/0021-9010.88.1.100 